\title{
Masses and thermodynamic properties of heavy mesons in the non-relativistic quark model using the Nikiforov-Uvarov method
}

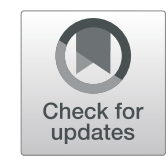

\author{
M. Abu-Shady, T. A. Abdel-Karim and Sh. Y. Ezz-Alarab
}

\author{
* Correspondence: dr.abushady@ \\ gmail.com \\ Department of Applied \\ Mathematics, Faculty of Science, \\ Menoufia University, Shebeen \\ El-Kom, Egypt
}

\begin{abstract}
Thermodynamic properties of heavy mesons are calculated within the framework of the N-dimensional radial Schrödinger equation. The Cornell potential is extended by including the quadratic potential plus the inverse of quadratic potential. The energy eigenvalues and the corresponding wave functions are calculated in the $\mathrm{N}$-dimensional space using the Nikiforov-Uvarov (NU) method. The obtained results are applied for

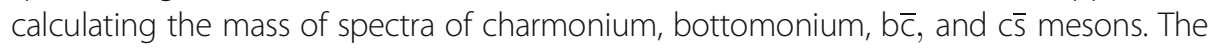
thermodynamic properties of heavy quarkonia such as the mean energy, the specific heat, the free energy, and the entropy are calculated. The effect of temperature and the dimensionality number on heavy meson masses and thermodynamic properties is investigated. The obtained results are improved in comparison with other theoretical approaches and in a good agreement with experimental data. We conclude that the present potential well describes thermodynamic properties in the three-dimensional space and also the higher dimensional space.
\end{abstract}

Keywords: Cornell potential, Thermodynamic properties, Schrödinger equation, Heavy mesons

\section{Introduction}

Thermodynamics is the branch of physics concerned in temperature and their relation to energy. This branch plays an important role in high energy physics [1]. According to statistical quantum chromodynamics (QCD), nuclear matter may undergo a color deconfined partonic phase, the quark-gluon plasma (QGP), at sufficiently high temperature and/or density. Over the past few decades, strenuous efforts have been made to devise clean and experimentally viable signals that can unambiguously identify the existence of QCD phase transition and trace out its signatures. Charmonium (a bound state of charm and anti-charm quarks) suppression had been predicted as a signature for the deconfinement transition $[2,3]$.

The Schrödinger equation (SE) plays an important role in describing many phenomena as in high energy physics. Thus, the solutions of the SE are important for calculating the mass of quarkonia and thermodynamic properties. To obtain the exact and approximate solutions of SE, various methods have been used for specific potentials such as the Nikiforov-Uvarov method [4], supersymmetry quantum mechanics [5], asymptotic iteration method [6], and Laplace integral transform [7]. Recently, $N$-dimensional

(c) The Author(s). 2019 Open Access This article is distributed under the terms of the Creative Commons Attribution 4.0 International License (http://creativecommons.org/licenses/by/4.0/), which permits unrestricted use, distribution, and reproduction in any medium provided you give appropriate credit to the original author(s) and the source, provide a link to the Creative Commons license, and indicate if changes were made. 
Schrödinger equation has received focal attention in the literature. The higher dimension studies facilitate a general treatment of the problem in such a manner that one can obtain the required results in the lower dimensions just dialing appropriate with $N$ [8-14].

The thermodynamic properties play an essential role in describing quark-gluon plasma [15], in which the thermodynamic properties of light quarks are calculated in comparison with the strange quark matter. In [16], the thermodynamic properties of the QGP are performed based on the constituent quasiparticle model of the quark-gluon plasma. In addition, the thermodynamic properties are investigated in the framework chiral quark models such as in [17-19] and also in molecular physics using the relativistic and nonrelativistic models [20-23].

In the present paper, we aim to calculate the $N$-dimensional Schrödinger equation analytically by using Nikiforov-Uvarov (NU) method firstly, and apply the present results to find the properties of quarkonium particles which are not considered in other works such as thermodynamic properties for charm matter.

The paper is organized as follows: In the "Theoretical description of the NikiforovUvarov method" section, the NU method is briefly explained. In the "The Schrödinger equation with the extended Cornell potential" section, the energy eigenvalues and the corresponding wave functions are calculated in the $N$-dimensional space. In the "Results and discussion" section, the results are discussed. In the "Summary and conclusion" section, the summary and conclusion are presented.

\section{Theoretical description of the Nikiforov-Uvarov method}

The NU method is briefly given that is a suitable method to obtain the solution of the second-order differential equation which has the following form:

$$
\Psi^{\prime \prime}(s)+\frac{\bar{\tau}(s)}{\sigma(s)} \Psi^{\prime}(\mathrm{s})+\frac{\tilde{\sigma}(s)}{\sigma(s)^{2}} \Psi(s)=0,
$$

where $\sigma(s)$ and $\tilde{\sigma}(s)$ are the polynomial of the maximum second degree and $\bar{\tau}(s)$ is a polynomial of the maximum first degree with a coordinate transformation $S=S(r)$ to find the particular solution of Eq. (1) by separation of variables, if we use the following transformation:

$$
\Psi(s)=\Phi(s) \chi(s) .
$$

Equation (1) can be written as in [23, 24] as follows:

$$
\begin{aligned}
& \sigma(s) \chi^{\prime \prime}(s)+\tau(s) \chi^{\prime}(s)+\lambda \chi(s)=0, \\
& \pi(s)=\frac{\sigma \prime(s)-\bar{\tau}(s)}{2} \pm \sqrt{\left(\frac{\sigma \prime(s)-\bar{\tau}(s)}{2}\right)^{2}-\tilde{\sigma}(s)+K \sigma(s),} \\
& \lambda=\lambda_{n}=-n \tau(s)^{\prime}-\frac{n(n-1)}{2} \sigma^{\prime \prime}(s), \quad n=0,1,2, \ldots
\end{aligned}
$$

$X(s)=X_{n}(s)$ is a polynomial of $n$ degree which satisfies the hypergeometric equation which has the following form: 


$$
\lambda=K+\pi^{\prime}(s)
$$

where $\pi(s)$ is a polynomial of the first degree. The values of $K$ in the square root of Eq. (4) is possible to calculate if the expressions under the square root are square of the expressions. This is possible if its discriminate is zero (for details, see [4]).

\section{The Schrödinger equation with the extended Cornell potential}

The SE for two particles interacting via symmetric potential in the $N$-dimensional space takes the form as in [25]

$$
\left[\frac{d^{2}}{d r^{2}}+\frac{N-1}{r} \frac{d}{d r}-\frac{L(L+N-2)}{r^{2}}+2 \mu(E-V(r))\right] \Psi(r)=0,
$$

where $L, N$, and $\mu$ are the angular momentum quantum number, the dimensionality number, and the reduced mass for the quarkonium particle, respectively. Setting wave function $\Psi(r)=r^{\frac{1-N}{2}} R(r)$, the following radial SE is obtained:

$$
\left[\frac{d^{2}}{d r^{2}}+2 \mu(E-V(r))-\frac{\left(L+\frac{N-2}{2}\right)^{2}-\frac{1}{4}}{2 \mu r^{2}}\right] R(r)=0,
$$

the extended Cornell potential is suggested as follows. $V(r)$ takes the form.

$$
V(r)=a r-\frac{b}{r}+c r^{2}+\frac{d}{r^{2}},
$$

where $a, b, c$, and $d$ are the arbitrary constants to be determined later. The first term is a linear term for confinement feature and coulomb's potential that describes the short distances between quark-antiquark. The two terms are called Cornell potential. In the present work, we extend the Cornell potential to include the quadratic potential and the inverse quadratic potential which play an important role in improving quarkonium properties such as in $[26,27]$.

By substituting Eq. (9) into Eq. (8), we obtain:

$$
\left[\frac{d^{2}}{d r^{2}}+2 \mu\left(E-a r+\frac{b}{r}-c r^{2}-\frac{d}{r^{2}}-\frac{\left(l+\frac{N-2}{2}\right)^{2}-\frac{1}{4}}{2 \mu r^{2}}\right)\right] R(r)=0,
$$

Let us assume that $r=\frac{1}{x}$ and $r_{0}$ is a characteristic radius of the meson. Then, the scheme is based on the expansion of $\frac{1}{x}$ in a power series around $r_{0}$, i.e., around $\delta=\frac{1}{r_{0}}$. Equation (10) takes the following equation:

$$
\left[\frac{d^{2}}{d x^{2}}+\frac{2 x}{x^{2}} \frac{d}{d x}+\frac{2 \mu}{x^{4}}\left(-A+B x-C_{1} x^{2}\right)\right] R(x)=0,
$$

where

$$
A=-\mu\left(E-\frac{3 a}{\delta}-\frac{6 a}{\delta^{2}}\right), \quad B=\mu\left(\frac{3 a}{\delta^{2}}+\frac{8 C}{\delta^{3}}+b\right),
$$

and 


$$
C_{1}=\mu\left(\frac{a}{\delta^{3}}+\frac{c}{\delta^{4}}-\frac{b}{\delta}+d+\frac{\left(l+\frac{N-2}{2}\right)^{2}-\frac{1}{4}}{2 \mu}\right),
$$

therefore,

$$
\pi= \pm \sqrt{\left(K+2 C_{1}\right) x^{2}-2 B x+2 A}
$$

the constant $K$ is chosen such as the function under the square root has a double zero, i.e., its discriminant for bound state solutions, we choose the positive sign in the above equation so that the derivative is given.

$$
\begin{aligned}
& \tau=2-\frac{2 B}{\sqrt{2 A}} \\
& \lambda=\lambda_{n}=-n \tau(s)-\frac{n(n-1)}{2} \sigma^{\prime \prime}(s), \quad n=0,1,2, \ldots
\end{aligned}
$$

and Eq. (14), we obtain:

$$
\lambda_{n}=-n\left(2-\frac{2 B}{2 \sqrt{A}}\right)-n(n-1)
$$

by using Eq. (5), we obtain from Eq. (6); $\lambda=\lambda_{n}$. The energy eigenvalue in the $N$-dimensional space is given.

$$
E_{n l}=\frac{3 a}{\delta}+\frac{6 c}{\delta^{2}}-\frac{2 \mu\left(\frac{3 a}{\delta^{2}}+\frac{8 c}{\delta^{3}}+b\right)^{2}}{\left[(2 n+1)+\sqrt{1+\frac{8 \mu a}{\delta^{3}}+4\left(\left(L+\frac{N-2}{2}\right)^{2}-\frac{1}{4}\right)-8 \mu d+\frac{24 \mu c}{\delta^{4}}}\right]^{2}},
$$

By taking $a=0$ and $N=3$, we obtain the results in [28]. At $d=0$, we obtain the results of [29].

The radial of the wave function of Eq. (10) takes the following form.

$$
R_{n l}=C_{n l} \frac{--B}{r^{\frac{-B}{2 A}}-1} e^{\sqrt{2 A} r}\left(-r^{2} \frac{d}{d r}\right)^{n}\left(r^{-2 n+\frac{-2 B}{\sqrt{2 A}}} e^{-2 \sqrt{2 A} r}\right),
$$

where $C_{n}$ is the normalization constant that is determined by:

$$
\int\left|R_{n l}(r)\right|^{2} d \boldsymbol{r}=1
$$

\section{Thermodynamic properties}

We study thermodynamic properties of extended Cornell potential; the partition function is given $Z=\sum_{n=0}^{[\lambda]} e^{-\beta E}$, where $\beta=\frac{1}{K T} ; K$ is the Boltzmann constant. The principal quantum number $n$ ranges from $0,1,2, \ldots,[\lambda], \lambda=\frac{1}{2}\left[\sqrt{\frac{A_{2}}{A_{1}}}-A_{3}\right]$ where $A_{1}, A_{2}, A_{3}$ are defined in Eq. (20). In the classical limit, at high temperature $T$ for large [ $\lambda]$, the sum can be replaced by an integral as used in $[20,21]$, in which the partition function and thermodynamic properties are defined as follows. 


\section{Partition function}

$$
Z(\beta)=\int_{0}^{\lambda} e^{-\beta E_{n}} d \lambda
$$

by substituting Eq. (16), we obtain:

$$
Z(\beta)=\frac{1}{2} e^{-A_{1} \beta}\left(-A_{3} e^{\frac{A_{2} \beta}{A_{3}^{2}}}+e^{\frac{A_{2} \beta}{\left(A_{3}+22\right)^{2}}}\left(A_{3}+2 \lambda\right)+\sqrt{A_{2}} \sqrt{\pi} \sqrt{\beta}\left(\operatorname{Erfi}\left[\frac{\sqrt{A_{2}} \sqrt{\beta}}{A_{3}}\right]-\operatorname{Erfi}\left[\frac{\sqrt{A_{2}} \sqrt{\beta}}{A_{3}+2 \lambda}\right]\right)\right),
$$

where

$$
\begin{aligned}
& A_{1}=\left(\frac{3 a}{\delta}+\frac{6 a}{\delta^{2}}\right), \quad A_{2}=2 \mu\left(\frac{3 a}{\delta^{2}}+\frac{8 C}{\delta^{3}}+b\right)^{2}, \\
& A_{3}=1+\sqrt{1+\frac{8 \mu a}{\delta^{3}}+4\left(\left(L+\frac{N-2}{2}\right)^{2}-\frac{1}{4}\right)-8 \mu d+\frac{24 \mu c}{\delta^{4}}}
\end{aligned}
$$

Mean energy $U$

$$
\begin{aligned}
U(\beta)=- & \frac{d}{d \beta} \operatorname{Ln} Z(\beta) . \\
U(\beta)=- & \left(2 \mathrm { e } ^ { A _ { 1 } \beta } \left(\frac{1}{2} e^{-A_{1} \beta}\left(-\frac{A_{2} e^{\frac{A_{2} \beta}{A_{3}}}}{A_{3}}+\frac{A_{2} \mathrm{e}^{\frac{A_{2} \beta}{\left(A_{3}+22\right)^{2}}}}{A_{3}+2 \lambda}+\sqrt{A_{2}} \sqrt{\pi} \sqrt{\beta} D+\frac{H}{2 \sqrt{\beta}}\right)\right.\right. \\
& \left.\left.-\frac{1}{2} A_{1} e^{-A_{1} \beta}\left(-A_{3} e^{\frac{A_{2} \beta}{A_{3}^{2}}}+e^{\frac{A_{2} \beta}{\left(A_{3}+2 \lambda\right)^{2}}}\left(A_{3}+2 \lambda\right)+\sqrt{\beta} H\right)\right)\right) \\
& \left./\left(-A_{3} e^{\frac{A_{2} \beta}{A_{3}}}+e^{\frac{A_{2} \beta}{\left(A_{3}+22\right)^{2}}}\left(A_{3}+2 \lambda\right)+\sqrt{\beta} H\right)\right),
\end{aligned}
$$

where

$$
\begin{aligned}
& D=\frac{\sqrt{A_{2}} e^{\frac{A_{2} \beta}{A^{2}}}}{A_{3} \sqrt{\pi} \sqrt{\beta}}-\frac{\sqrt{A_{2}} e^{\frac{A_{2} \beta}{\left(A_{3}+2 \lambda\right)^{2}}}}{\sqrt{\pi} \sqrt{\beta}\left(A_{3}+2 \lambda\right)}, \\
& H=\operatorname{Erfi}\left[\frac{\sqrt{A_{2}} \sqrt{\beta}}{A_{3}}\right]-\operatorname{Erfi}\left[\frac{\sqrt{A_{2}} \sqrt{\beta}}{A_{3}+2 \lambda}\right],
\end{aligned}
$$

\section{Specific heat $C$}

$$
C(\beta)=\frac{d U}{d T}=-K \beta^{2} \frac{d U}{d \beta}
$$




$$
\begin{aligned}
& C(\beta)=-K \beta^{2}\left(\left(2 e ^ { A _ { 1 } \beta } \left(\frac{1}{2} e^{-A_{1} \beta}\left(-\frac{A_{2} e^{\frac{A_{2} \beta}{A_{3}{ }^{2}}}}{A_{3}}+\frac{A_{2} e^{\frac{A_{2} \beta}{\left(A_{3}+2 \lambda\right)^{2}}}}{A_{3}+2 \lambda}+\sqrt{A_{2}} \sqrt{\pi} \sqrt{\beta} D+\frac{\sqrt{A_{2}} \sqrt{\pi} H}{2 \sqrt{\beta}}\right)\right.\right.\right. \\
& -\frac{1}{2} A_{1} \mathrm{e}^{-A 1 \beta}\left(-A_{3} e^{\frac{A_{2} \beta}{A_{3}{ }^{2}}}+e^{\frac{A_{2} \beta}{\left(A_{3}+2 l\right)^{2}}}\left(A_{3}+2 \lambda\right)+\sqrt{A_{2}} \sqrt{\pi} \sqrt{\beta} H\right) \\
& \left(-\frac{A_{2} e^{\frac{A_{2} \beta}{A_{3}{ }^{2}}}}{A_{3}}+\frac{A_{2} e^{\frac{A_{2} \beta}{\left(A_{3}+2 \lambda\right)^{2}}}}{A_{3}+2 \lambda}+\sqrt{A_{2}} \sqrt{\pi} \sqrt{\beta} D+\frac{\sqrt{A_{2}} \sqrt{\pi} H}{2 \sqrt{\beta}}\right) \\
& \left.\left.\left.+e^{\frac{A_{2} \beta}{\left(A_{3}+2 \lambda\right)^{2}}}\left(A_{3}+2 \lambda\right)+\sqrt{A_{2}} \sqrt{\pi} \sqrt{\beta} H\right)\right)\right) / \\
& /\left(-A_{3} \frac{\mathbb{A}_{2} \beta}{\mathbb{A}^{2}{ }^{2}}+\square^{\frac{A_{2} \beta}{\left(A_{3}+2 \lambda\right)^{2}}}\left(A_{3}+2 \lambda\right)+\sqrt{A_{2}} \sqrt{\pi} \sqrt{\beta} H\right)
\end{aligned}
$$

\section{Free energy}

$$
\begin{aligned}
& F(\beta)=-K T \operatorname{LnZ}(\beta), \\
& F(\beta)=-\frac{1}{\beta} \log \left[\frac{1}{2} \mathrm{e}^{-\mathrm{A}_{1} \beta}\left(-A_{3} e^{\frac{A_{2} \beta}{a_{3}^{2}}}+e^{\frac{A_{2} \beta}{\left(A_{3}+21\right)^{2}}}\left(A_{3}+2 \lambda\right) \sqrt{A_{2}} \sqrt{\pi} \sqrt{\beta}\left(\operatorname{Erfi}\left[\frac{\sqrt{A_{2}} \sqrt{\beta}}{A_{3}}\right]-\operatorname{Erfi}\left[\frac{\sqrt{A_{2}} \sqrt{\beta}}{A_{3}+2 \lambda}\right]\right)\right)\right] .
\end{aligned}
$$

\section{Entropy}

$$
\begin{aligned}
S(\beta)= & K \ln \mathrm{Z}(\beta)+K T \frac{\partial}{\partial T} \ln \mathrm{Z}(\beta) . \\
S(\beta)= & K \beta^{2}\left(-\left(2 e ^ { A _ { 1 } \beta } \left(\frac { 1 } { 2 } e ^ { - A _ { 1 } \beta } \left(-\frac{A_{2} e^{\frac{A_{2} \beta}{4_{3}}}}{A_{3}}+\frac{A_{2} e^{\frac{A_{2} \beta}{\left.A_{3}+21\right)^{2}}}}{A_{3}+2 \lambda}+\sqrt{A_{2}} \sqrt{\pi} \sqrt{\beta} D-\frac{1}{2} A_{1} \mathrm{e}^{-A 1 \beta}\right.\right.\right.\right. \\
& \left.\left.\left(-A_{3} e^{\frac{A_{2} \beta}{A_{3}}}+e^{\frac{A_{2} \beta}{\left(A_{3}+21\right)^{2}}}\left(A_{3}+2 \lambda\right)+\sqrt{A_{2}} \sqrt{\pi} \sqrt{\beta} H\right)\right)\right) \\
& /\left(\left(\beta\left(-A_{3} e^{\frac{A_{2} \beta}{A_{3}^{2}}}+e^{\frac{A_{2} \beta}{\left(A_{3}+2 \lambda\right)^{2}}}\left(A_{3}+2 \lambda\right)+\sqrt{A_{2}} \sqrt{\pi} \sqrt{\beta} H\right)\right)\right. \\
& \left.\left.+\sqrt{A_{2}} \sqrt{\pi} \sqrt{\beta} H\right)\right)-\frac{1}{\beta} \log \left[\frac{1}{2} e^{-A_{1} \beta}\left(-A_{3} e^{\frac{A_{2} \beta}{e^{2}}}+e^{\frac{A_{2} \beta}{\left(A_{3}+2 \lambda\right)^{2}}}\left(A_{3}+2 \lambda\right)+\sqrt{A_{2}} \sqrt{\pi} \sqrt{\beta} H\right)\right],
\end{aligned}
$$

\section{Results and discussion}

\section{Quarkonium masses}

In this section, we calculate the spectra of the heavy quarkonium system such as charmonium and bottomonium that have the quark and antiquark flavor; the mass of quarkonium is calculated in 3-dimensional space $(N=3)$. So, we apply the following relation as in [23-26].

$$
M=2 m+E_{n l},
$$


where $m$ is the bare quark mass for quarkonium. By using Eq. (16), we can write Eq. (25) as follows:

$$
\begin{aligned}
M= & 2 m+\frac{3 a}{\delta} \\
& +\frac{6 c}{\delta^{2}}-\frac{2 \mu\left(\frac{3 a}{\delta^{2}}+\frac{8 c}{\delta^{3}}+b\right)^{2}}{\left[(2 n+1)+\sqrt{1+\frac{8 \mu a}{\delta^{3}}+4\left(\left(L+\frac{N-2}{2}\right)^{2}-\frac{1}{4}\right)-8 \mu d+\frac{24 \mu c}{\delta^{4}}}\right]^{2}}
\end{aligned}
$$

In Table 1, we calculated the energy eigenvalue from $1 \mathrm{~S}$ to $2 \mathrm{D}$. The charmonium mass is calculated by using Eq. (26). The free parameters of the present calculations are $a, b, c$, and $\delta$ and are fitted with experimental data. In addition, quark masses are obtained from [28]. In Table 1, we note that the calculated masses of charmonium are in good agreement with experimental data and are improved in comparison with $[8,25$, $28,30,31$ ], in which maximum error in comparison experimental data is about 0.0555 . The effect of dimensional number plays an important role in recent works [7-11]. The general form of higher dimensional gives more information about the system under study. In addition, we note that the charmonium mass increases with the increasing dimensional number due to the increasing binding energy. Therefore, binding energy is larger than the constituents of charmonium which give us the limitation of nonrelativistic models. The effect is also studied in [11].

In Table 2, we note the present results for bottomonium are in agreement with experimental data, in which maximum error equals 0.0004828 and the present results are improved in comparison with $[8,25,28,31]$, in which different potential and methods are used. We note that the effect of dimensionality has the same effect as charmonium.

In Table 3, we calculate the mass spectra of b $\bar{c}$ mesons where $2 m=m_{b}+m_{c}$ in Eq. (26). We find that the $1 \mathrm{~S}$ state closes with the experimental data. The experimental data of the other states are not available. Hence, the theoretical predictions of the

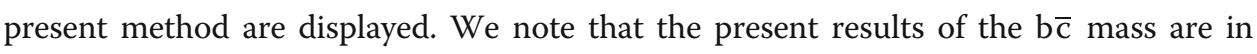
good agreement in comparison with [32, 35, 36].

In Table 4, we calculate the mass spectra of $\mathrm{c} \overline{\mathrm{s}}$ mesons where $2 m=m_{c}+m_{s}$ in Eq. (26). The $1 \mathrm{~S}, 2 \mathrm{~S}$, and $1 \mathrm{D}$ are close with experimental data and are improved in comparison with power potential, screened potential, and phenomenological potential

Table 1 Mass spectra of charmonium (in GeV) $\left(m_{c}=1.209 \mathrm{GeV}, a=0.01 \mathrm{GeV}^{2}, b=14.94\right.$, $\left.d=-15.04 \mathrm{GeV}^{-1}, c=0.02 \mathrm{GeV}^{3}, \delta=1.7 \mathrm{GeV}\right)$

\begin{tabular}{lllllllll}
\hline State & Present work & {$[8]$} & {$[28]$} & {$[30]$} & {$[25]$} & {$[31]$} & $N=4$ & Exp. [32] \\
\hline $1 \mathrm{~s}$ & 3.095 & 3.078 & 3.096 & 3.096 & 3.096 & 3.078 & 3.360 & 3.096 \\
1p & 3.258 & 3.415 & 3.433 & 3.433 & 3.255 & 3.415 & 3.673 & - \\
$2 \mathrm{~s}$ & 3.685 & 4.187 & 3.686 & 3.686 & 3.686 & 3.581 & 3.698 & 3.686 \\
1D & 3.510 & 3.752 & 3.767 & 3.770 & 3.504 & 3.749 & 3.895 & - \\
2p & 3.779 & 4.143 & 3.910 & 4.023 & 3.779 & 3.917 & 3.827 & 3.773 \\
3s & 4.040 & 5.297 & 3.984 & 4.040 & 4.040 & 4.085 & 3.966 & 4.040 \\
$4 \mathrm{~s}$ & 4.262 & 6.407 & 4.150 & 4.355 & 4.269 & 4.589 & 3.986 & 4.263 \\
2D & 3.928 & - & - & 3.096 & - & 3.078 & 4.170 & 4.159 \\
\hline
\end{tabular}


Table 2 Mass spectra of bottomonium (in GeV) ( $m_{b}=4.823 \mathrm{GeV}, a=0.798 \mathrm{GeV}^{2}, b=5.051$,

\begin{tabular}{|c|c|c|c|c|c|c|c|c|}
\hline State & Present work & [8] & [28] & [31] & [25] & [33] & $N=4$ & Exp. [34] \\
\hline $1 \mathrm{~s}$ & 9.460 & 9.510 & 9.460 & 9.460 & 9.460 & 9.510 & 9.610 & 9.460 \\
\hline $1 p$ & 9.609 & 9.862 & 9.840 & 9.811 & 9.619 & 9.862 & 10.022 & - \\
\hline $2 s$ & 10.022 & 10.627 & 10.023 & 10.023 & 10.023 & 10.038 & 10.072 & 10.023 \\
\hline 1D & 9.846 & 10.214 & 10.140 & 10.161 & 9.864 & 10.214 & 10.205 & - \\
\hline $2 p$ & 10.109 & 10.944 & 10.160 & 10.374 & 10.114 & 10.396 & 10.269 & - \\
\hline $3 \mathrm{~s}$ & 10.360 & 11.726 & 10.280 & 10.355 & 10.355 & 10.566 & 10.306 & 10.355 \\
\hline $4 s$ & 10.580 & 12.834 & 10.420 & 10.655 & 10.567 & 11.094 & 10.344 & 10.580 \\
\hline
\end{tabular}

in [36]. The effect of dimensionality number has the same effect as in the charmonium and bottomonium.

In Fig. 1, we note that the partition function $Z$ decreases with increasing $\beta$. The range of $\beta=4.0$ to $6.5 \mathrm{GeV}^{-1}$ corresponding to $T=0.154$ to $0.250 \mathrm{GeV}$ represents the range of temperature which charmonium melts to its constituents as charm quark. We note that the partition function decreases with increasing $\beta$ and the dimensionality number $N$ (Fig. 2). In addition, we note the partition function is not sensitive at the largest values of $\beta$. The behavior of the partition function will act on other observables that will be discussed.

In [20], it is observed that the partition function $Z$ decreases monotonically with increasing $\beta$ in which the author applied the deformed five-parameter exponential-type potential in the SE. In [21], the partition function decreases with increasing $\beta$. Therefore, the behavior of $Z$ is in agreement with [20,21].

In Fig. 3, we note that the $U$ decreases with increasing of $\beta$ and increases with increasing of $\lambda$. In Fig. 4, the values of $U$ shift to higher values by increasing dimensionality number. In [21], the authors found that the internal energy increases with increasing $\lambda$ for HCL. In [20], the internal energy decreases with increasing $\lambda$. In [22], the authors calculated all thermodynamic properties of a neutral particle in a magnetic cosmic string background of using the non-relativistic SchrödingerPauli equation, in which they found that internal energy increases with increasing temperature and angular quantum number. We obtained the same conclusion in the present work for internal energy. Thus, the behavior of internal energy is in agreement with recent works [20-22]. In Fig. 4, we note that the $U$ increases with increasing dimensionality number. This effect is not considered in other works such as in $[20-22]$.

Table 3 Mass spectra of $b \bar{c}$ meson (in GeV) $\left(m_{b}=4.823 \mathrm{GeV}, m_{c}=1.209 \mathrm{GeV}, a=0.606 \mathrm{GeV}^{2}\right.$, $\left.b=3.651, d=-2.199 \mathrm{GeV}^{-1}, c=0.1 \mathrm{GeV}^{3}, \delta=1.2 \mathrm{GeV}\right)$

\begin{tabular}{lllllll}
\hline State & Present work & {$[32]$} & {$[35]$} & {$[36]$} & $N=4$ & Exp. [34] \\
\hline $1 \mathrm{~s}$ & 6.277 & 6.349 & 6.264 & 6.270 & 6.355 & 6.277 \\
$1 \mathrm{p}$ & 7.042 & 6.715 & 6.700 & 6.699 & 6.883 & - \\
$2 \mathrm{~s}$ & 7.383 & 6.821 & 6.856 & 6.835 & 6.878 & - \\
$2 \mathrm{p}$ & 6.663 & 7.102 & 7.108 & 7.091 & 7.161 & - \\
$3 \mathrm{~s}$ & 7.206 & 7.175 & 7.244 & 7.193 & 8.035 & - \\
\hline
\end{tabular}


Table 4 Mass spectra of $c \bar{s}$ meson in $(\mathrm{GeV})\left(m_{c}=1.628, m_{s}=0.419 \mathrm{GeV}, a=0.48 \mathrm{GeV}^{2}, b=3.795\right.$,

\begin{tabular}{lllllll}
\multicolumn{6}{l}{$\left.d=-1.481 \mathrm{GeV}^{-1}, c=0.1 \mathrm{GeV}^{3}, \delta=1.6 \mathrm{GeV}\right)$} \\
\hline State & Present work & Power & Screened & Phenomenological & $N=4$ & Exp. \\
\hline 1s & 1.968 & 1.9724 & 1.9685 & 1.968 & 2.3001 & 1968.3 [33] \\
1p & 2.565 & 2.540 & 2.7485 & 2.566 & 2.742 & - \\
2s & 2.709 & 2.6506 & 2.8385 & 2.815 & 2.797 & $2.709[37]$ \\
3S & 2.932 & 2.9691 & 3.2537 & 3.280 & 2.967 & - \\
1D & 2.857 & - & - & - & 3.934 & $2.859[37]$ \\
\hline
\end{tabular}

In Fig. 5, we note that the specific heat $(C)$ decreases with increasing $\beta$ and it shifts to higher values by increasing parameter $\lambda$. In addition, the dimensionality number shifts the specific heat to higher values as in Fig. 6. The behavior of the specific head is studied in [20-22] using the Schrödinger equation. We found the qualitative agreement with these works.

In Fig. 7, we note that the free energy $F$ increases with increasing $\beta$ and $\lambda$. Also, the free energy increases with increasing $N$, in Fig. 8. In [15], the quark-gluon plasma is assumed to be composed of the light quarks only such as the up and down quarks, which interact weakly, and the gluons which are treated as they are free. They found the free energy decreases with increasing temperature. In [38], the authors studied free energy for strange quark matter and they found the conclusion of [15]. In [22], the authors calculated the free energy of a neutral particle and found the free energy decreases with increasing temperature. In the present work, we note charm quark matter is qualitative agreement with $[15,22,38]$.

In Figs. 9 and 10, the entropy decreases with increasing $\beta$. In addition, the entropy shifts to higher values by increasing $N$ and $\lambda$. In [15, 22, 37], the authors found the entropy increases with increasing temperature for light quark, strange quark, and natural particles. We have the same conclusion for charm quark. In addition, we found there is no changing in the phase transition in the interval of temperatures that indicate

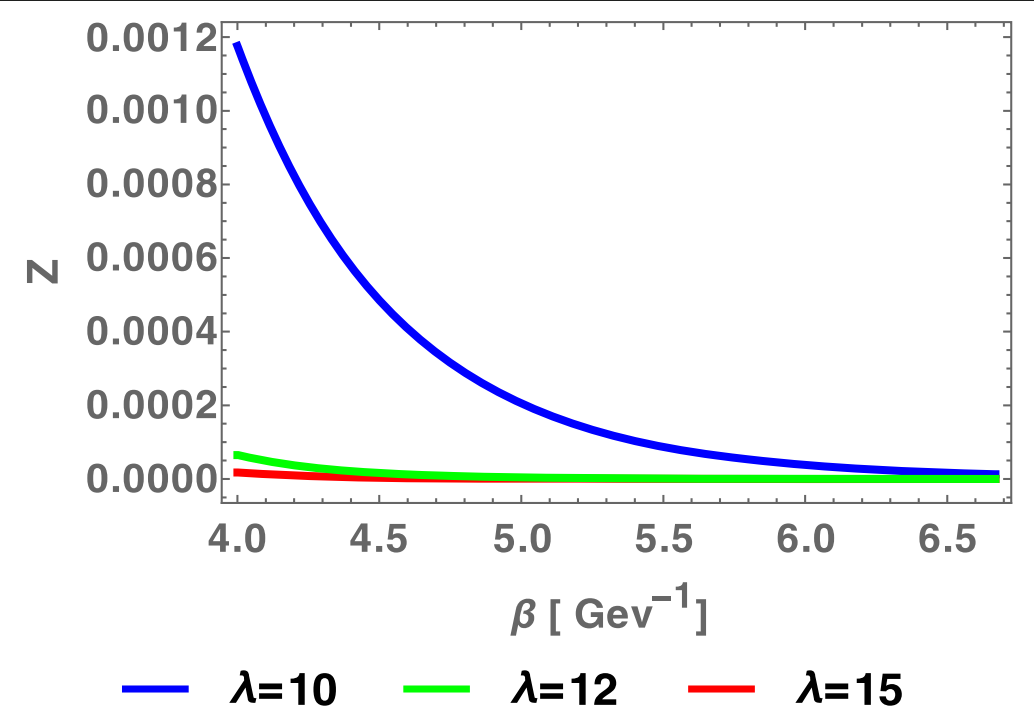

Fig. 1 Partition function is plotted as a function of $\beta$ for different values of $\lambda$ 


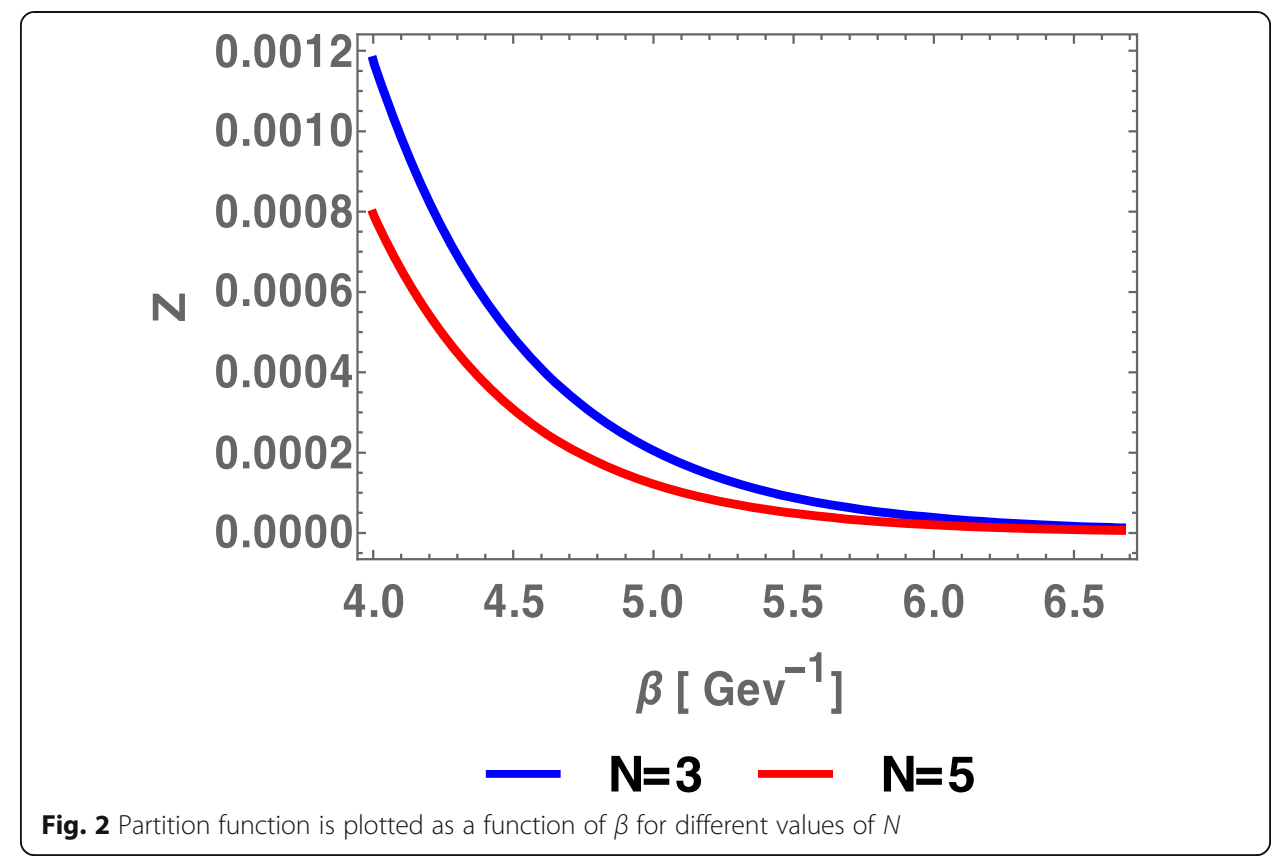

the charmonium melts below the critical temperature. In [39], the author shows that the dissociation of charmonium is found at below critical temperature.

\section{Summary and conclusion}

In the present work, the energy eigenvalues and the wave functions are obtained in the $N$-dimensional space by solving the $N$-radial Schrödinger equation using the NU method. The Cornell potential is extended to include the quadratic potential and
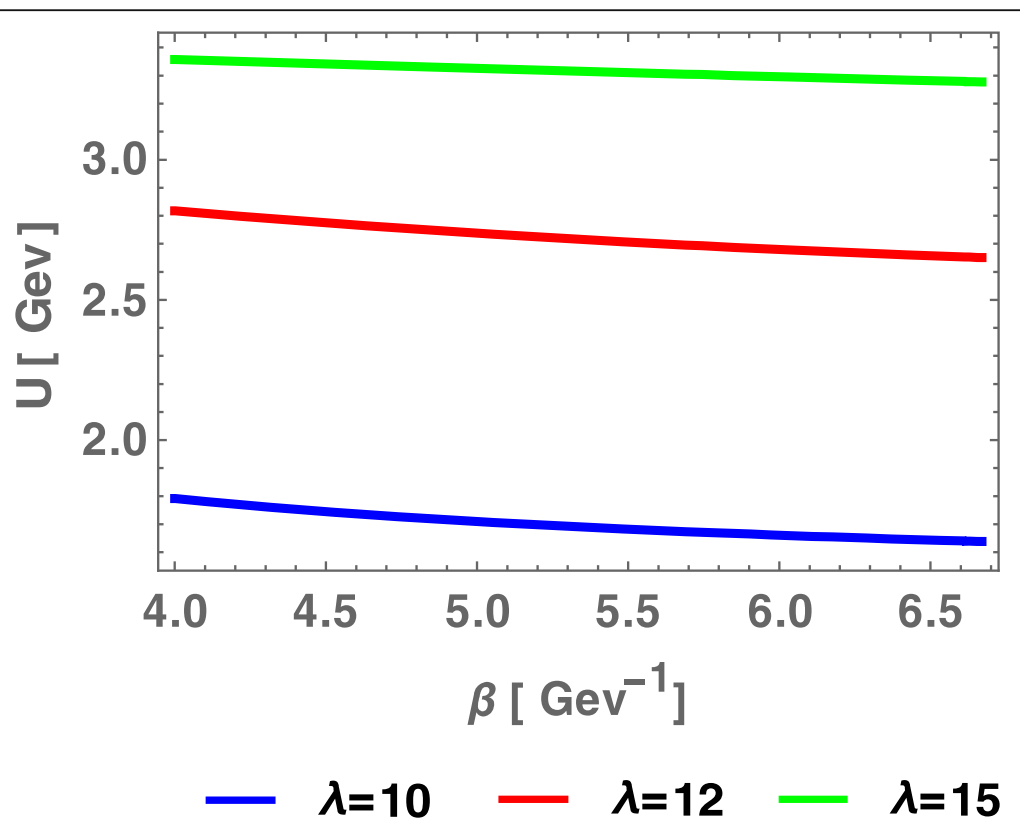

Fig. 3 Internal energy $U$ against $\beta$ for different values of $\lambda$ 


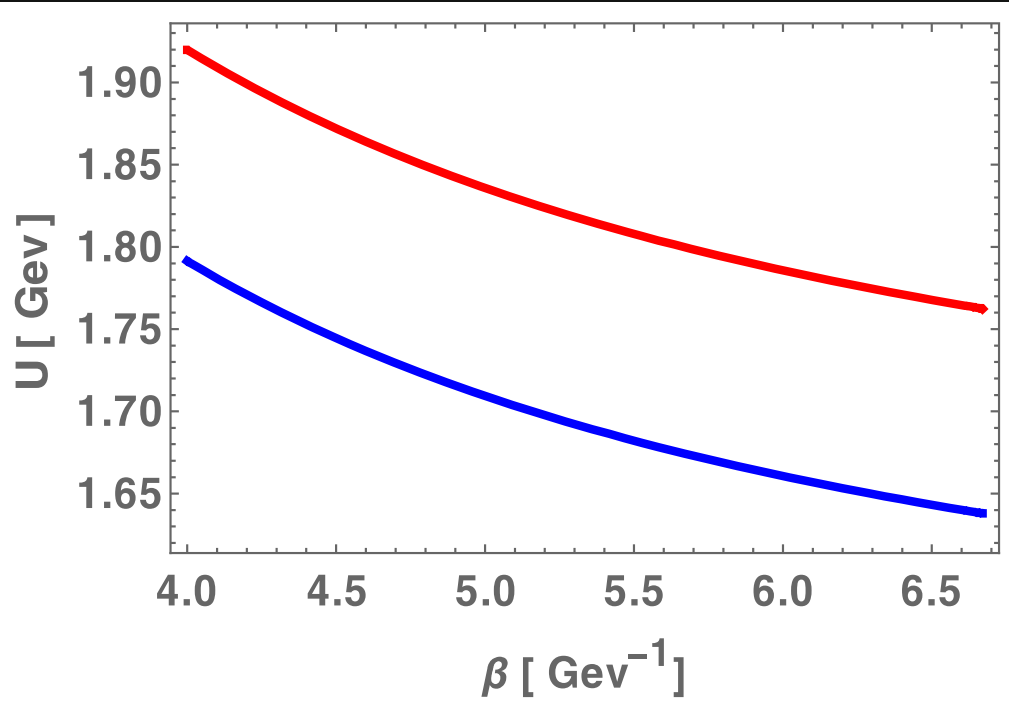

$-\mathrm{N}=3-\mathrm{N}=5$

Fig. 4 Internal energy $U$ against $\beta$ for different values of $N$

inverse quadratic potential which give a good description of heavy meson. We obtained the energy eigenvalues and wave functions in the higher dimensional space. In addition, special cases are obtained from the general form of the eigenvalue of energy. We applied the present results to calculate heavy meson masses and thermodynamic properties.

We conclude the following points:

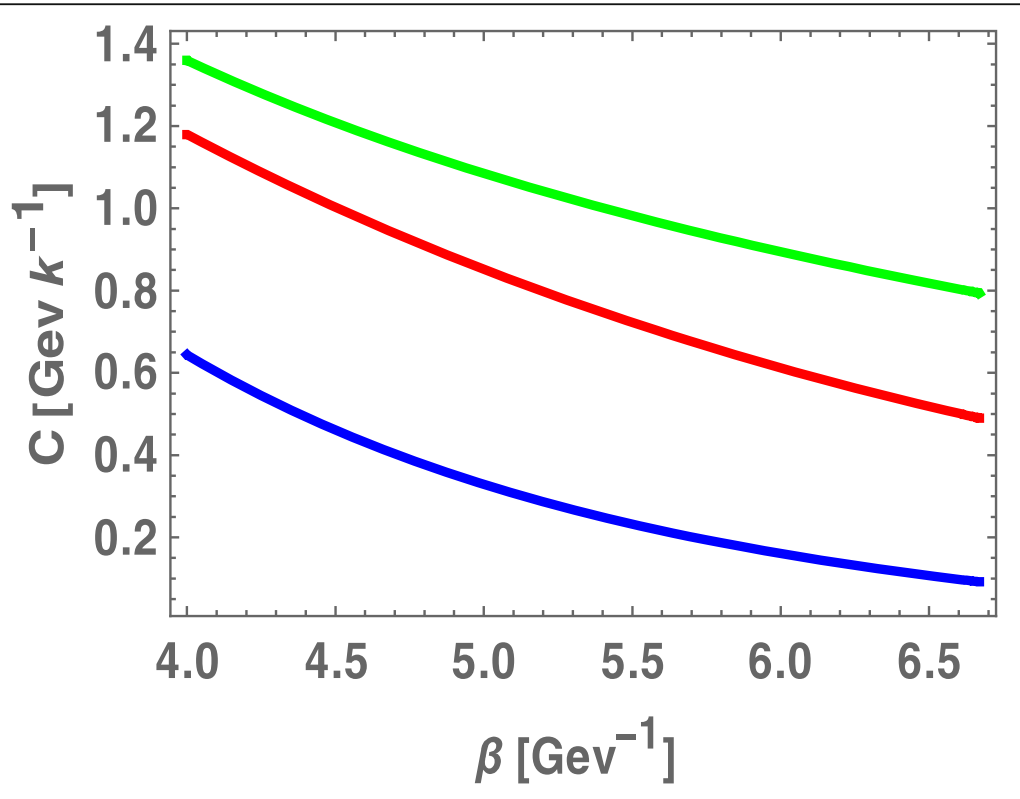

$-\lambda=10-\lambda=12-\lambda=15$

Fig. 5 Specific heat $C$ is plotted against $\beta$ different values of $\lambda$ 


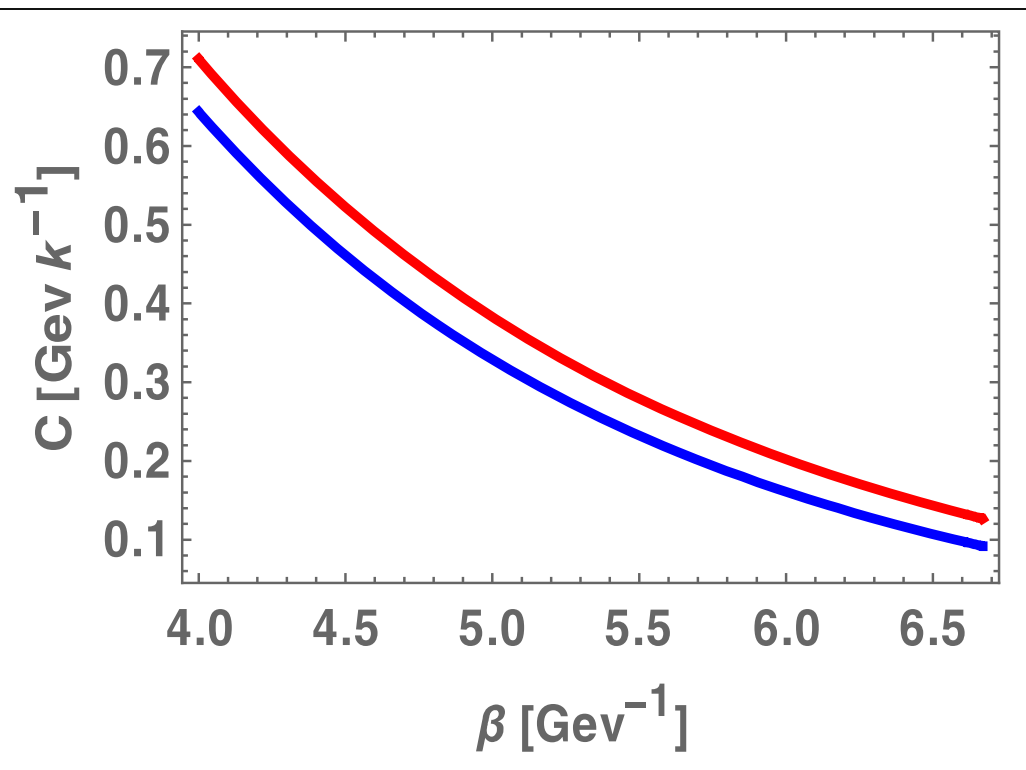

$-\mathrm{N}=3-\mathrm{N}=5$

Fig. $\mathbf{6}$ Specific heat $C$ is plotted against $\beta$ for different values of $N$

I-At $N=3$, the heavy meson masses are calculated. We found that the present results are in good agreement in comparison with experimental data. We found that the maximum errors are 0.0555 for the charmonium, 0.0004828 for the bottomonium, 0

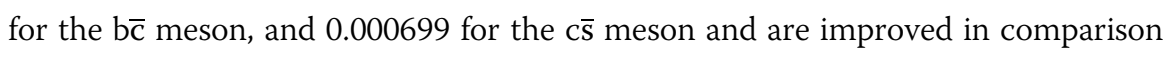
with other published works that used other potentials or techniques. For higher dimensional space $(N>3)$, we noted that the mass of spectra of charmonium,

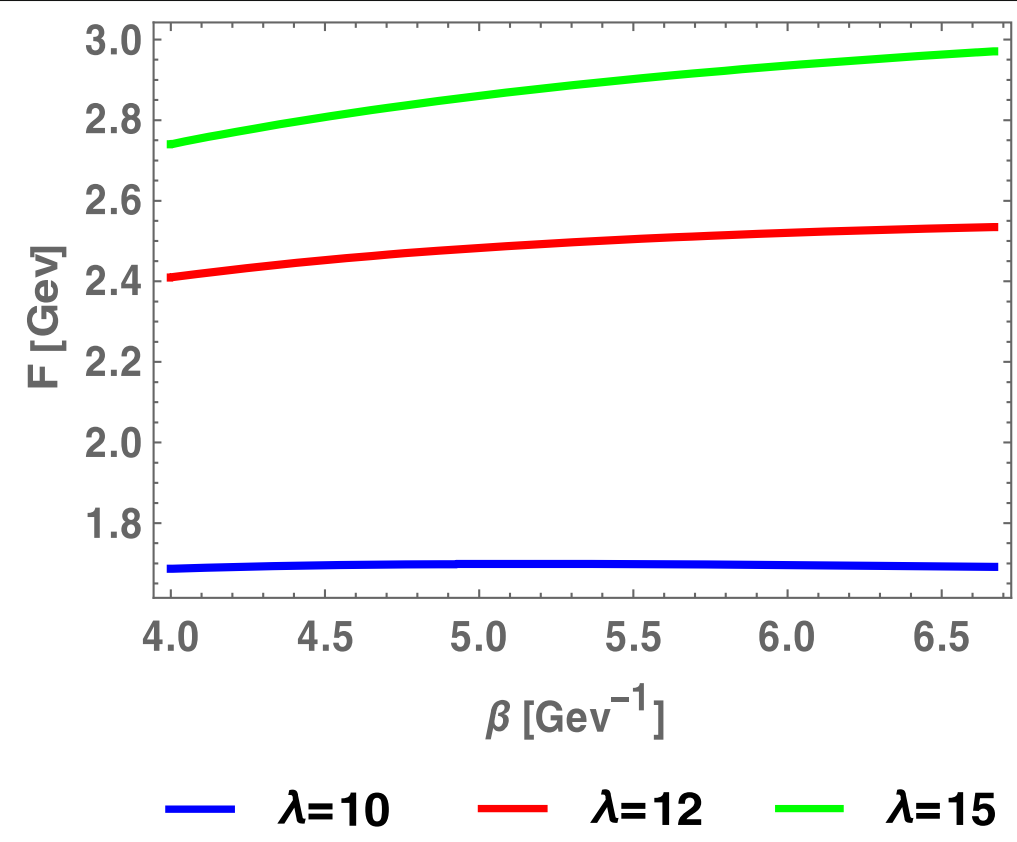

Fig. 7 Free energy $F$ is plotted against $\beta$ for different values of 


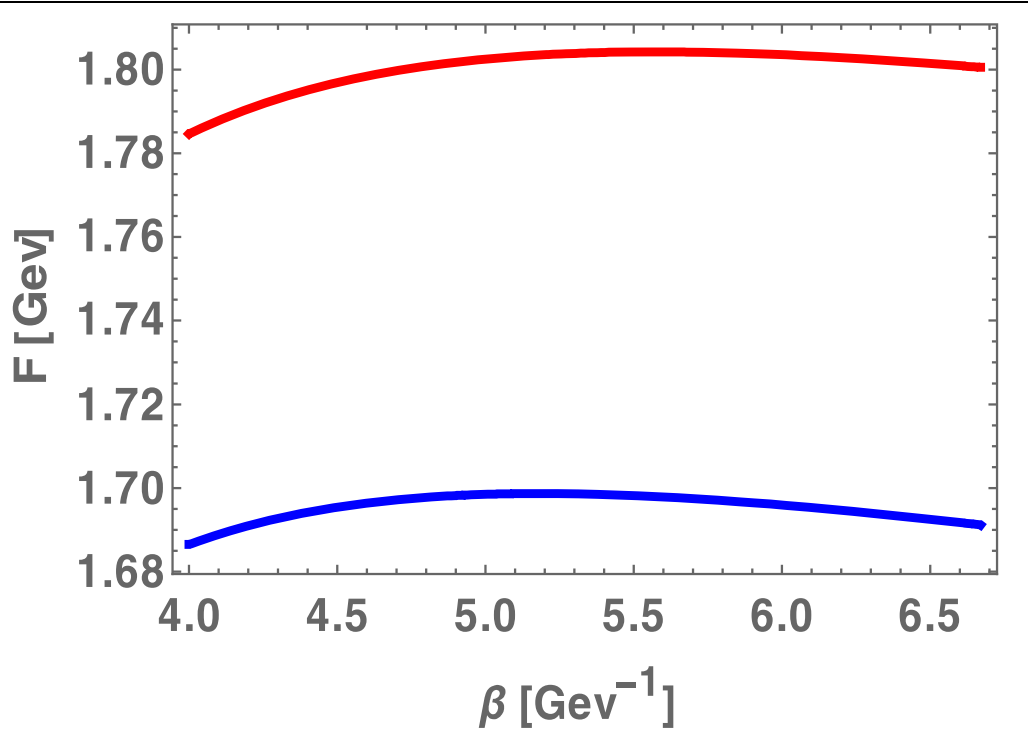

Fig. 8 Free energy $F$ is plotted against $\beta$ for different values of $N$

bottomonium, b $\bar{c}$, and $\mathrm{c} \overline{\mathrm{s}}$ mesons increase with the increasing dimensional number due to the increase in the corresponding binding energy. The binding energy nears to the constituents of heavy meson masses (charm quark). Thus, the velocity of charm quark closes to the velocity of light which leads to the limitation of non-relativistic quark models when $N$ takes larger values.

II-At $N=3$, the thermodynamic properties are calculated such as internal energy, free energy, specific heat, and entropy. We found the internal energy, the specific heat, and the entropy decrease with increasing $\beta$. In $[15,22,37]$, thermodynamic properties

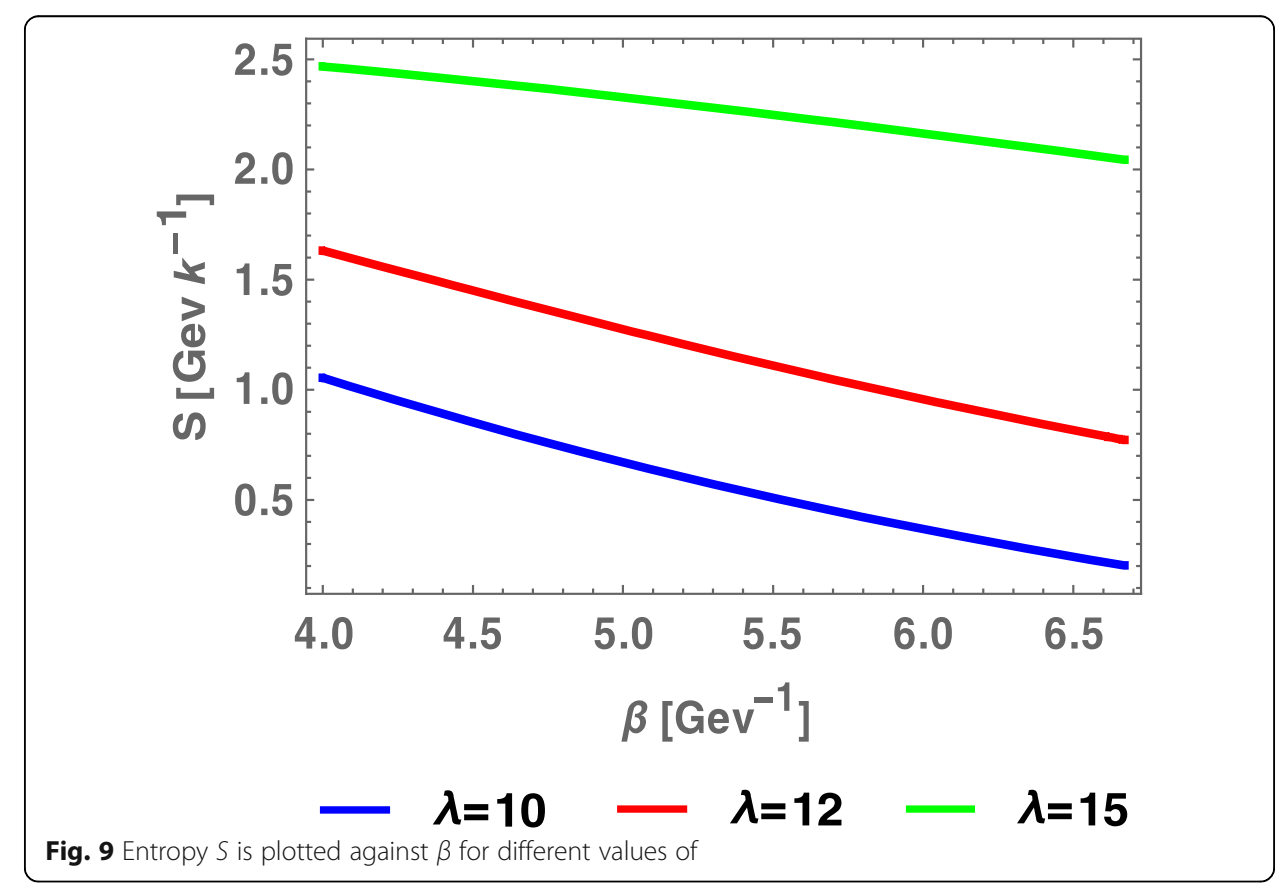




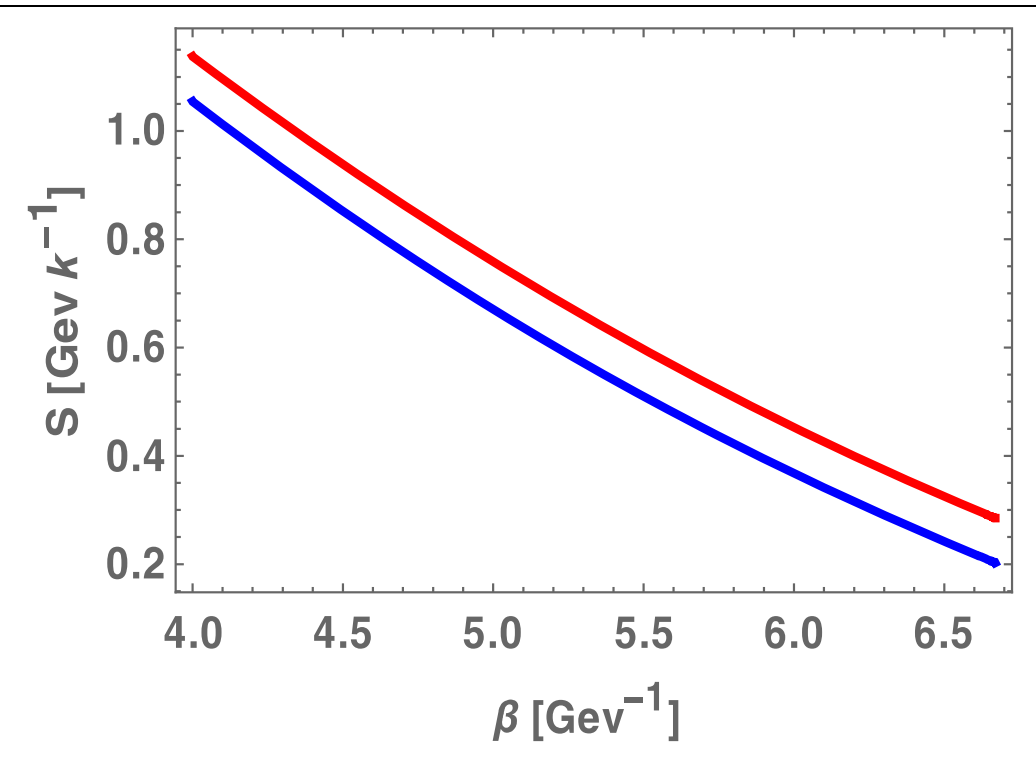

Fig. 10 Entropy $S$ is plotted against $\beta$ for different values of $N$

for the light quark, the strange quark, and natural particles are studied. The present results are in qualitative agreement with $[15,22,37]$. So far, the thermodynamic properties of charmonium are not considered in many works such as [15, 22, 37]. For $N>3$, the dimensionality number plays an important role in changing the behavior of thermodynamic properties for charm matter. We noted from the figures that the internal energy, the free energy, the specific heat, and the entropy shift to higher values by increasing dimensional number. Therefore, the dimensional number increases the energetic of charm matter. We hope to extend this work to include spin-spin interaction and spin-orbital interaction. In addition, the effect of external magnetic field on heavy meson properties gives more information about quark-gluon plasma as a future work.

Acknowledgements

The authors think the reviewers for their comments that supported this work.

Authors' contributions

MA-S suggested the point research and follows up the steps of calculations and reviewed it. TAAK participates in carrying out the calculations of the results and reviewed it. ShYE-A carried out the calculations and wrote it. All authors read and approved the final manuscript.

Authors' information

Not applicable

Funding

Not applicable

Availability of data and materials

All data generated during this study are included in the list of references in the paper. 
Received: 26 March 2019 Accepted: 7 May 2019

Published online: 18 June 2019

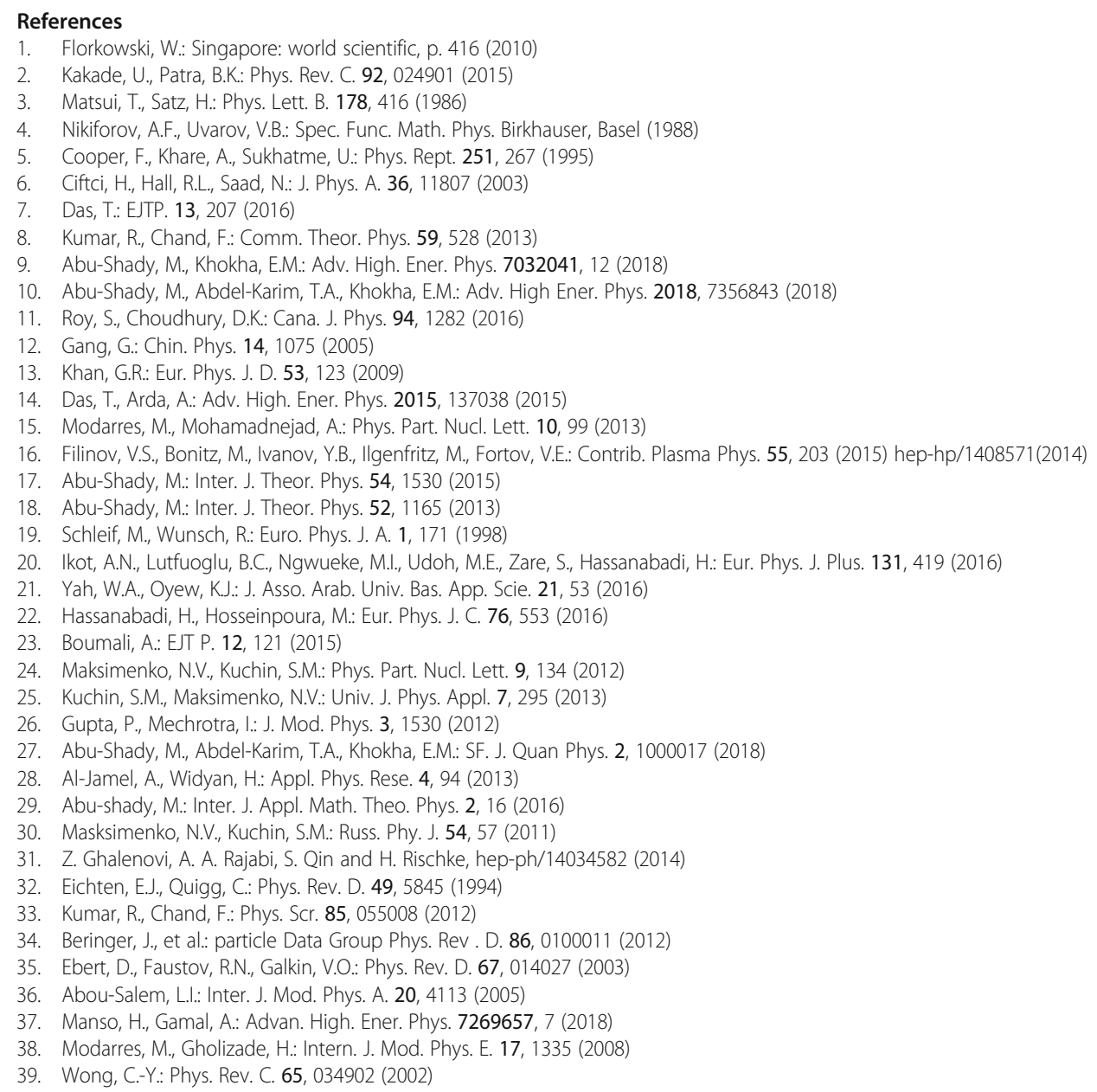

\section{Publisher's Note}

Springer Nature remains neutral with regard to jurisdictional claims in published maps and institutional affiliations.

\section{Submit your manuscript to a SpringerOpen ${ }^{\circ}$ journal and benefit from:}

- Convenient online submission

- Rigorous peer review

- Open access: articles freely available online

High visibility within the field

- Retaining the copyright to your article 